\title{
Paramètres de solubilité
}

\section{Clef du succès en formulation !}

\section{Patrick de LANTY}

RHODIA Recherches \& Technologies Centre de Recherches et Technologies $d^{\prime}$ Aubervilliers (CRTA), Pôle Formulation et Revêtement. Laboratoire Applicabilité 52, rue de la Haie coq F - 93308 Aubervilliers CEDEX. Télécopie : 33 (0) 149376302 <Patrick.de-Lanty@eu.rhodia.com>

La méthode des paramètres de solubilité a été mise au point il y a maintenant près de quarante ans par Charles M. Hansen (membre de la Danish Academy of Technical Sciences). II travaillait à l'époque dans un laboratoire de recherche danois pour l'industrie des peintures et vernis. Cette méthode est l'aboutissement d'un long travail théorique et pratique pour comprendre et expliquer un milieu difficile à modéliser, l'état liquide, qui se situe entre deux « limites " : I'état gazeux où les molécules sont très mobiles mais éloignées les unes des autres, et l'état solide où les molécules sont très proches mais pratiquement immobiles. «Des trois états usuels de la matière, gaz, liquide et solide cristallin, l'état liquide a été le dernier à être étudié quantitativement et il est encore le moins bien compris » $[1,2,9]$.

\section{Comment formuler ?}

Tous ceux qui travaillent dans le domaine de la formulation savent combien il est parfois difficile de solubiliser ce que I'on appelle en jargon de métier "un caillou ». Lorsqu'un solide (le soluté) n'est pas soluble dans l'eau, il reste deux possibilités : I'une consiste à le réduire en poudre afin de le disperser dans ce milieu avec un système tensioactif. On obtient une dispersion à peu près stable si les tensioactifs sont bien choisis et la poudre suffisamment fine. Mais, pour différentes raisons, cette option n'est pas toujours satisfaisante. L'autre consiste à trouver un solvant du « caillou » pour obtenir une solution homogène.

Là les problèmes commencent! Quel solvant choisir? Il y en a des centaines sinon des milliers ! De quelle famille chimique? Avec quelles propriétés physiques? Pendant longtemps la seule technique connue a été la méthode dite

\begin{abstract}
The Hansen solubility parameter method, whilst undoubtedly an important advance academically was, in practice, little used. However this method can save considerable time in developing formulations in many different kinds of industrial activities.

The way of formulating an active chemical is often difficult. Is it better to choose an aqueous or non aqueous solution, with one solvent or several? Is an emulsion preferable? What to do to formulate several solutes in the same system?

The main principles are established before studying the applications: how to choose a single solvent or a solvent blend, how to "create" a new solvent with well defined properties, the choice of an oil to make an emulsion or how to formulate several solutes in a single formulation.

Then the limits of the method are explored.
\end{abstract}

Key words: emulsion, formulation, Hansen, Hildebrand, solubility, volume

« de l'étagère » qui consistait à essayer patiemment les solvants les uns après les autres, plus ou moins au hasard. L'expérience et l'intuition de l'expérimentateur permettant de compenser l'absence de méthode scientifique. Que dire quand il faut dissoudre dans le même solvant plusieurs solides de propriétés physicochimiques complètement différentes. II faut une bonne dose d'optimisme en n'étant, au final, pas sûr que le résultat trouvé soit le meilleur et même qu'il y ait un résultat acceptable! C'est dans tous les cas beaucoup de temps perdu.

\section{La méthode : principes de base}

Avec les paramètres de solubilité, le formulateur dispose d'une méthode simple et rapide pour arriver au meilleur résultat sans gaspillage de temps!

Il existe au sein des molécules quatre types de forces de cohésion : les trois forces de Van der Waals (interactions de London, de Keesom et de Debye) et la force de liaison hydrogène. Ces forces permettent à un solvant de rester à l'état liquide. Elles s'opposent efficacement à l'agitation thermique. Si l'on chauffe le liquide, il arrive un moment où l'énergie apportée au système devient juste suffisante pour rompre les forces de liaisons qui maintiennent les molécules au contact et le solvant passe à l'état gazeux. Cette énergie $\Delta H v$ est appelée enthalpie de vaporisation. Pour avoir une valeur correcte de l'énergie « $E$ » des forces existant entre les molécules, il faut soustraire la valeur du travail effectué pour éloigner les molécules les unes des autres lors du changement d'état. Elle est donnée par la loi des gaz parfaits $\mathrm{PV}=\mathrm{RT}$ pour 1 mole de gaz.
On a donc l'énergie de cohésion molaire du système $E=\Delta H_{v}-R T$.

À partir de cette base thermodynamique, Joël Hildebrand (1881-1983) a introduit le concept de paramètre de solubilité dans des travaux théoriques développés pendant la première moitié du vingtième siècle.

Il définit le paramètre de solubilité global d'une substance comme étant la racine carrée de l'énergie de cohésion par unité de volume, avec $\mathrm{V}$ le volume molaire, soit :

$$
\delta=\sqrt{\left(\Delta H_{v}-R T\right) / V}
$$

En l'état, ce paramètre $\delta$ est de peu d'utilité pratique.

Charles M. Hansen $[3-5,7,8]$, profitant du développement de l'informatique dans les années 1960, a eu l'idée de scinder ce paramètre « global » en trois de ses composantes principales: les forces dites de "dispersion » de London $\boldsymbol{\delta}_{\mathbf{D}}$, les forces de polarité de Keesom $\boldsymbol{\delta}_{\mathbf{P}}$ (entre dipôles permanents), et les forces de liaison hydrogène $\boldsymbol{\delta}_{\mathbf{H}}$. Les forces de Debye toujours faibles en valeurs absolues (dipôles induits) sont négligées en première approximation.

Hansen obtient ainsi un espace à trois dimensions dans lequel toutes les substances liquides ou solides peuvent être localisées en supposant que l'énergie totale de cohésion des dites substances est la somme géométrique des énergies intermoléculaires définies plus haut, de telle sorte que l'on peut écrire :

$$
\delta=\sqrt{\delta_{D}{ }^{2}+\delta_{p}^{2}+\delta_{H}^{2}}
$$

De manière pratique, on calcule pour chaque liquide les valeurs des paramètres $\boldsymbol{\delta}_{\mathbf{D}}, \boldsymbol{\delta}_{\mathbf{p}}$ et $\boldsymbol{\delta}_{\mathbf{H}}$ puis on porte ces grandeurs selon les trois axes de cet espace. Le point correspondant repré- 


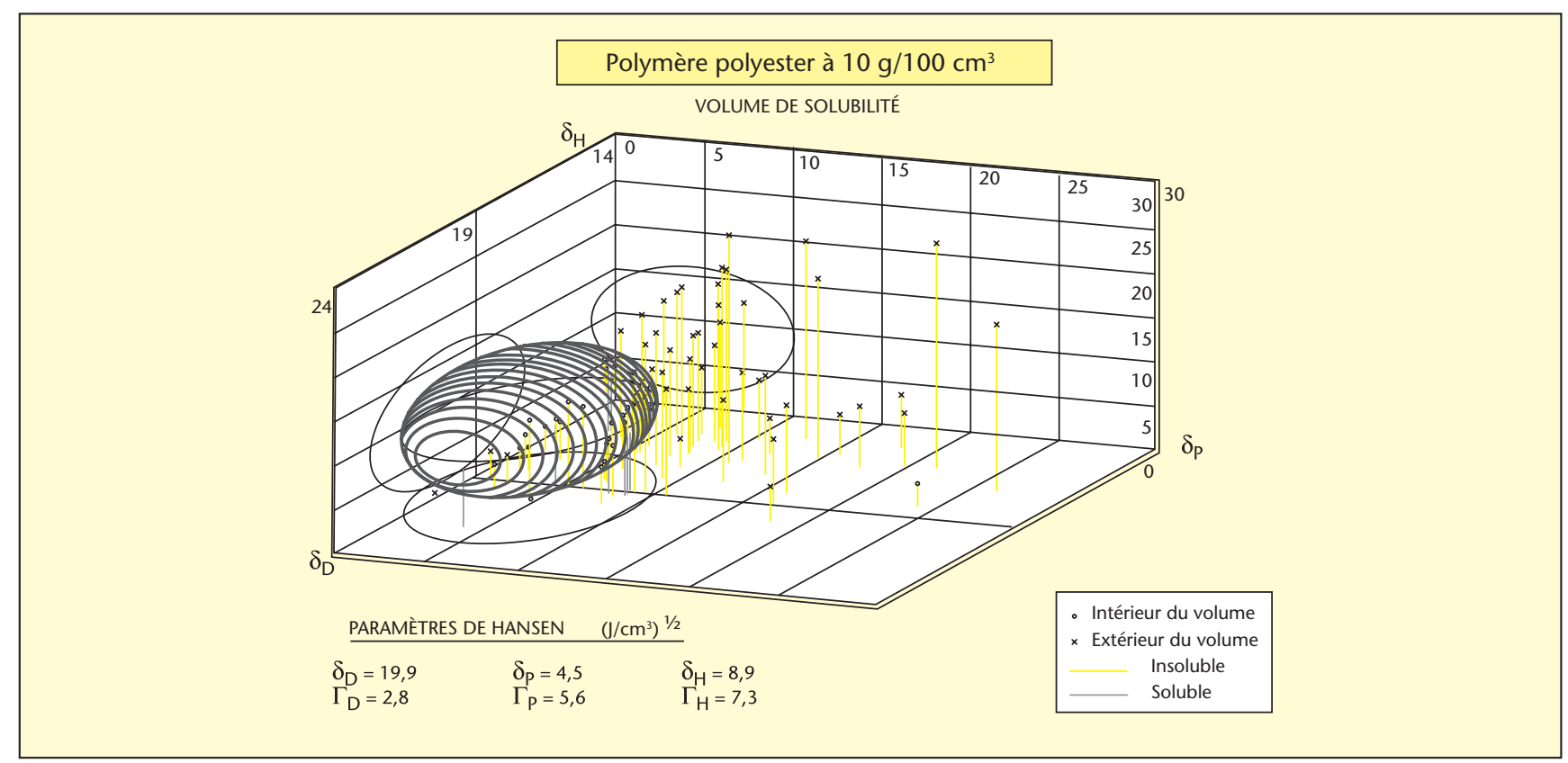

Figure 1. Exemple pour un polymère commercial.

sente la combinaison des interactions qui assure la cohésion du liquide [9].

Pour qu'une substance solide quelconque soit soluble dans un liquide, ou que deux liquides soient miscibles entre eux, il faut que leur situation dans l'espace soit voisine, c'est-à-dire que leurs paramètres de solubilité soient proches. La puissance de la méthode vient de la possibilité de travailler sur les mélanges de solvants sachant que les paramètres de solubilité d'un mélange sont proportionnels aux fractions volumiques des constituants du mélange.

Sans entrer dans les détails théoriques, le mot " proche » signifie que le soluté et le solvant, ou mélange de solvants sont suffisamment voisins pour que l'enthalpie de mélange " $\Delta H_{M}$ " (terme positif qui s'oppose à la dissolution) ne soit pas supérieure au facteur associant l'entropie et la température : « $T \Delta S$ » (terme négatif qui favorise la dissolution).

En effet, pour qu'il y ait dissolution, la variation d'enthalpie libre « $\Delta \mathrm{G}$ » doit toujours être négative.

C'est la loi de Gibbs-Helmholtz : $\Delta \mathrm{C}=\Delta H_{M}-T \Delta S$

\section{Le volume de solubilité}

En conséquence, un soluté occupe le centre d'un volume de solubilité dans lequel la fonction $\Delta \mathrm{G}$ est négative. Tous les solvants et mélanges se trouvant dans ce volume sont solvants vrais du soluté en question. Ceux qui se trouvent à l'extérieur sont des non-solvants (voir exemple à la figure 1, pour un polymère commercial).

Pour déterminer les paramètres de solubilité d'un soluté, il suffit de le mélanger avec une série standard de solvants bien répartis dans l'espace de Hansen, de calculer le $\boldsymbol{\delta}$ global de Hildebrand, et de préciser les limites du volume par quelques mélanges de solvants et de non solvants. Ces tests se prêtent bien à la robotisation.

\section{Les applications pratiques}

Elles sont nombreuses et intéressent tous les secteurs de l'industrie.

\section{Choix d'un solvant}

On peut calculer la position dans ou hors du volume de n'importe quel solvant non testé dont on connaît les paramètres et ainsi en prévoir l'efficacité. II est possible de trier les solvants potentiels par ordre de distance au centre du volume. Les plus proches du centre, donc du soluté, sont ceux qui thermodynamiquement ont le plus de chance de donner une solution stable.

\section{Mélange de deux non-solvants}

Beaucoup plus spectaculaire : si l'on choisit de faire un mélange de deux non-solvants situés de part et d'autre du volume, afin que les paramètres du mélange soit dans le volume, la composition sera solvante ! II faut cependant que ces deux liquides ne soient pas trop éloignés pour éviter une démixtion.

\section{Choix d'un mélange}

Plus simplement, s'il n'existe pas de solvant dans la zone recherchée, on peut calculer à partir de solvants existant sur le marché, et sélectionnés en fonction de critères physicochimiques, économiques et environnementaux, une formulation de deux ou plusieurs constituants ayant les mêmes paramètres que le solvant idéal.

\section{Création d'un solvant vert!}

Chaque groupe d'atomes dans une molécule contribue à la valeur finale des trois paramètres $\delta_{\mathbf{D}}, \boldsymbol{\delta}_{\mathbf{p}}$ et $\boldsymbol{\delta}_{\mathbf{H}}$. II est possible par modélisation $d^{\prime}$ 'une nouvelle molécule chimique, de remplacer un solvant efficace, mais ayant certains inconvénients (prix, liquide trop volatil, inflammable, nocif, etc.) par une autre substance ayant les mêmes propriétés solvantes mais sans en avoir les inconvénients.

Ceci dit, le choix des éléments devant constituer le meilleur solvant pour l'application comme pour l'environnement n'est pas évident. L'idée même du solvant « vert » est souvent très subjective. La plupart du temps, un produit « vert » est assimilé à un produit extrait des plantes ou dont la molécule est proche de celle de substances naturelles. Or, c'est une idée trop simple, sinon simpliste. Le pétrole dérive de végétaux! La caféine extraite de plantes (café, thé, cacao) est classée nocive par ingestion (R22). Certains produits toxiques sont mêmes nécessaires à la vie à très faibles doses, ainsi la vitamine D3 (cholécalciférol). De fait, la nocivité est souvent plus une question de quantité que de qualité.

Il y a quelques années, le d-limonène, solvant puissant extrait de l'écorce d'agrumes, a été industrialisé pour remplacer des solvants de 
synthèse jugés nocifs. Solvant naturel, il a pris des parts de marché non négligeables jusqu'au moment où il a été classé irritant et très toxique pour l'environnement (R 50/53)!

II n'y a donc pas de recette universelle. Pratiquement, on utilise les éléments constituants la molécule comme des briques à assembler en sélectionnant celles qui sont connues comme ne présentant en général pas de risque. Certaines fonctions (ester, cétone, alcool...) sont préférables, d'autres sont à éviter, les azotés en particulier (amine, nitrile...). On peut partir d'une molécule naturelle sans danger et la modifier pour lui donner les propriétés recherchées. Les acides gras servent souvent de base de départ mais ils donnent des solvants à haut point d'ébullition. Les esters de diacides donnent également de bons solvants biodégradables et plus polyvalents. L'élaboration de solvants de faible masse moléculaire est moins évidente. Leur pression de vapeur est élevée et il est difficile de ne pas avoir d'effet sur le système respiratoire et sur les muqueuses, sauf à choisir des éléments n'ayant aucune interaction avec l'organisme (silicones, perfluorés, etc.). Cependant, nous avons pu remplacer, en suivant cette procédure, un solvant toxique classé « CMR » par un éther-ester aux caractéristiques très voisines mais dépourvu de nocivité et sans danger pour l'environnement.

\section{Choix d'une " huile » pour émulsion}

Les huiles végétales ou animales sont généralement des triglycérides d'acides gras. Par extension, on appelle « huile » tout solvant plus ou moins visqueux et de point d'ébullition élevé. L'huile sélectionnée en fonction de ses paramètres de solubilité peut servir de solvant d'un principe actif insoluble dans l'eau. II suffit ensuite de disperser l'huile dans l'eau (émulsion directe) avec les tensioactifs appropriés.

\section{Formulation de plusieurs solutés}

La méthode permet également de résoudre des problèmes plus complexes où l'on doit formuler plusieurs solutés dans une même composition solvante. Par exemple, avec deux produits solides, il suffit que les volumes des deux substances aient une zone commune (intersection des deux ensembles) et de calculer une composition de solvants dont les paramètres sont situés dans ce domaine. Ce type de problème est classique en santé humaine, en santé animale, en agrochimie, etc.

Un laboratoire doit par exemple formuler plusieurs principes actifs insolubles dans l'eau dans une seule composition liquide. L'eau doit être le véhicule majoritaire. L'émulsion s'impose. Mais comment procéder?

Une étude des paramètres de solubilité de chacun des solutés permet de sélectionner l'huile ou une association d'huiles afin de dissoudre la quantité optimale de chacun d'entre eux. Ce problème réglé, il reste à trouver l'association de tensioactifs pour stabiliser l'émulsion.

Un autre avantage, et non des moindres: on peut très rapidement affirmer qu'un problème n'a pas de solution. Par exemple, si deux solutés ont des volumes disjoints!

\section{Les limites de la méthode}

- Elle ne s'applique pas aux non-électrolytes, c'est-à-dire en particulier aux minéraux et aux sels, substances dans lesquelles les liaisons sont fortement ioniques.

- L'impasse faite sur les liaisons de Van der Waals de type Debye conduit à une certaine proportion d'anomalies (de l'ordre de 10 à $20 \%$ ). Cela veut dire qu'un liquide situé dans le volume de solubilité n'est pas toujours solvant. Ce risque est d'autant plus élevé que l'on se rapproche de la zone limite du volume.

- Avec un système simplifié à trois paramètres, la visualisation du volume est facilitée mais la méthode ne permet qu'une évaluation qualitative : le liquide solubilise ou non la substance à dissoudre. Pour avoir un calcul quantitatif, donc connaître la concentration maximale possible avec les substances organiques solides, il faut intégrer les quatre types de forces intermoléculaires et tenir compte des effets acidobasiques au sens de Lewis. Ce travail a été initié par Rhône Poulenc au début des années 1990 avec un succès prometteur, mais demanderait un travail plus poussé de type universitaire pour être publié.

Les approximations introduites par Hansen peuvent en partie expliquer pourquoi sa méthode à trois paramètres a suscité, au départ, peu d'enthousiasme parmi les théoriciens. Par contre, en milieu industriel, sa facilité d'utilisation et les résultats qu'elle permet d'obtenir dans un minimum de temps ont fait son succès, en particulier dans le domaine des peintures non aqueuses $[6,9]$. Ce constat explique qu'elle soit maintenant reconnue et enseignée dans les établissements universitaires.

\section{RÉFÉRENCES}

1. HILDEBRAND J. SCOTT. The solubility of nonelectrolytes. New York : Reinhold, 1949.

2. BURRELL $H$. The challenge of the solubility parameter concept. JPT $1968 ; 40(520)$.

3. HANSEN CM. The three dimensional solubility parameter. Key to paint component affinities. I. Solvents, plasticizers, polymers and resins. JPT ; 39(505).

4. HANSEN CM. The three dimensional solubility parameter : II Dyes, emulsifiers, mutual solubility and compatibility and pigments. JPT 1967 ; 39(511).

5. HANSEN CM, SKAARUP K. III. Independent calculation of the parameter components. JPT $1967 ; 39(511)$.

6. BAGLEY EB, NELSON TP, SCIGLIANO JM. Three-dimensional Solubility Parameters and their relationship to internal pressure measurements in polar and hydrogen bonding solvents. JPT 1971 ; 43(555).

7. HANSEN CM, BEERBOWER A. Solubility parameters. Kirk Othmer. Sup vol. $2^{e}$ édition, 1971, p. 889-910.

8. Hansen CM. Hansen solubility parameters : A User's Handbook. CRC Press, 2000.

9. CABANE B, HENON S. Liquides. Paris: Belin, Collection Echelles, 2003. 